\title{
Focal Rosai-Dorfman disease coexisting with lymphoma in the same anatomic site: a localized histiocytic proliferation associated with MAPK/ERK pathway activation
}

\author{
Sofia Garces ${ }^{1} \cdot$ C. Cameron Yin ${ }^{1} \cdot$ Keyur P. Patel ${ }^{1} \cdot$ Joseph D. Khoury $\mathbb{D}^{1} \cdot$ John T. Manning Jr. ${ }^{1}$ - Shaoying Li $\mathbb{D}^{1}$ \\ Jie Xu (i) ${ }^{1} \cdot$ Sergio Pina-Oviedo ${ }^{2} \cdot$ Malisha R. Johnson ${ }^{1} \cdot$ Sergio González ${ }^{3} \cdot$ Montserrat Molgó $^{4}$. \\ Roberto Ruiz-Cordero $\mathbb{1}^{1} \cdot$ L. Jeffrey Medeiros ${ }^{1}$
}

Received: 4 June 2018 / Revised: 3 September 2018 / Accepted: 4 September 2018 / Published online: 15 October 2018

(c) United States \& Canadian Academy of Pathology 2018

\begin{abstract}
Rosai-Dorfman disease is a rare histiocytic disorder shown to have gene mutations that activate the MAPK/ERK pathway in at least one-third of cases. Most patients with Rosai-Dorfman disease present with bulky lymphadenopathy or extranodal disease, but rarely Rosai-Dorfman disease is detected concomitantly with lymphoma in the same biopsy specimen. The underlying molecular mechanisms of focal Rosai-Dorfman disease occurring in the setting of lymphoma have not been investigated. We report 12 cases of Rosai-Dorfman disease and lymphoma involving the same anatomic site. There were five men and seven women (age, 23 to 77 years) who underwent lymph node $(n=11)$ or skin $(n=1)$ biopsy; the lymphomas included nodular lymphocyte predominant Hodgkin lymphoma $(n=6)$, classical Hodgkin lymphoma $(n=4)$, small lymphocytic lymphoma $(n=1)$ and extranodal marginal zone lymphoma $(n=1)$. The foci of Rosai-Dorfman disease in all cases had S100 protein-positive histiocytes undergoing emperipolesis. No patients had Rosai-Dorfman disease at other anatomic sites at initial diagnosis and at last follow-up (median, 40 months). We performed immunohistochemical analysis to assess activity of the MAPK/ERK pathway in the Rosai-Dorfman disease foci. We also micro-dissected disease foci and analyzed 146 genes using next-generation sequencing in four cases with adequate DNA; the panel included genes previously reported to be mutated in Rosai-Dorfman disease. All cases were negative for gene mutations. Nevertheless, all cases were positive for cyclin D1 and most cases showed p-ERK expression indicating that the MAPK/ERK pathway is active in the histiocytes of focal Rosai-Dorfman disease. We conclude that focal Rosai-Dorfman disease coexisting with lymphoma is a clinically benign and localized histiocytic proliferation. These data also indicate that the MAPK/ERK pathway is active in focal Rosai-Dorfman disease although we did not identify activating mutations. These findings suggest that the pathogenesis of focal Rosai-Dorfman disease is different from that of usual cases of Rosai-Dorfman disease.
\end{abstract}

L. Jeffrey Medeiros

ljmedeiros@mdanderson.org

1 Department of Hematopathology, The University of Texas MD Anderson Cancer Center, Houston, TX, USA

2 Department of Pathology, University of Arkansas for Medical Sciences, Little Rock, AR, USA

3 Department of Pathology, Facultad de Medicina, Pontificia Universidad Católica de Chile, Santiago, Chile

4 Department of Dermatology, Facultad de Medicina, Pontificia Universidad Católica de Chile, Santiago, Chile

\section{Introduction}

Rosai-Dorfman disease is a rare histiocytic disorder in which lymph nodes and/or extranodal sites are infiltrated by abnormal histiocytes with round to oval nuclei, dispersed chromatin, distinct central nucleoli, and abundant pale or vacuolated cytoplasm, some of which demonstrate emperipolesis $[1,2]$. Emperipolesis, a hallmark of Rosai-Dorfman disease, is defined as the active, non-destructive engulfment of leukocytes including lymphocytes, plasma cells, and granulocytes by histiocytes [3]. Most patients with Rosai-Dorfman disease present with bulky lymph nodes or pseudotumor-like extranodal sites of disease. Rarely, Rosai-Dorfman disease occurs in patients with lymphoma. This association can occur simultaneously or at different 
times and most often involves separate anatomic sites [4-18]. A small subset of patients, however, has foci of Rosai-Dorfman disease and lymphoma involving the same biopsy specimen. In this scenario, Rosai-Dorfman disease is most often an incidental histologic finding, and patients do not have the disease involving other sites [5, 10-12, 15].

In the largest next-generation sequencing-based genomic study to date, mutually exclusive KRAS and MAP2K1 mutations were identified in approximately one-third of patients with Rosai-Dorfman disease [19]. Also, p-ERK overexpression was detected by immunohistochemistry in all cases harboring MAP2K1 mutation, indicating that mutations in Rosai-Dorfman disease activate the mitogenactivated protein kinase/extracellular regulated kinase (MAPK/ERK) signaling pathway [19]. Others have reported similar mutations and additional NRAS and $A R A F$ mutations in smaller series and case reports [20-23]. Overall, these findings suggest that mutated cases of Rosai-Dorfman disease are neoplastic and that these mutations can be targeted for therapy, as shown by others $[2,21]$.

The biology of cases of focal Rosai-Dorfman disease coexistent with lymphoma remains unknown. It has been postulated that Rosai-Dorfman disease in this setting might be secondary to an underlying local immune dysregulation, perhaps induced by the presence of lymphoma $[6,10]$. However, these studies were conducted before the role of the MAPK/ERK pathway in the pathogenesis of Rosai-Dorfman disease became known.

In this study, we report the clinicopathologic features of 12 cases of focal Rosai-Dorfman disease coexisting with lymphoma in the same biopsy specimen. We also performed immunohistochemistry to assess the activation status of the MAPK/ERK pathway and we assessed four cases for gene mutations using next-generation sequencing methods. To our knowledge, this is the first study that evaluates the underlying biology, including mutation status, of focal Rosai-Dorfman disease occurring concomitantly with lymphoma.

\section{Materials and methods}

\section{Case selection}

The study group is composed of 12 cases of Rosai-Dorfman disease associated with lymphoma in the same anatomic location. A total of 11 cases with available paraffin blocks or unstained slides of excisional biopsy specimens were retrieved from the archives of the Department of Hematopathology at The University of Texas MD Anderson Cancer Center from 1 January 1998 through 31 January 2017. In addition, a paraffin block of one additional case was obtained from the archives of the Department of
Pathology of the Pontificia Universidad Católica de Chile, Santiago, Chile. Available clinical and laboratory data were retrieved from the medical records. This study was approved by the Institutional Review Board at the University of Texas MD Anderson Cancer Center and carried out in accord with the Declaration of Helsinki.

\section{Histologic evaluation}

We reviewed hematoxylin-eosin-stained slides for all cases and the lymphomas were classified according to current World Health Organization criteria. Rosai-Dorfman disease was defined as aggregates of abnormal large histiocytes with round or oval nuclei, dispersed chromatin, visible nucleoli, and abundant pale cytoplasm with some histiocytes showing emperipolesis of lymphocytes, plasma cells, and/or granulocytes. The Rosai-Dorfman disease foci were measured in each sample using an Olympus glass eyepiece (WH10X/22) and $\times 20$ magnification.

\section{Immunohistochemistry}

Immunohistochemical studies were performed using formalin-fixed, paraffin-embedded tissue sections as described previously [24]. The antibody panels for each case were variable according to lymphoma type and differences over time. The antibodies used included reagents specific for: CD1a and CD21 (Leica Biosystem, Newcastle, UK); CD2, CD3, CD4, CD7, CD8, CD20, CD30, CD45/LCA, CD68, CD163, Bcl-6, and MUM1/IRF4 (DAKO, Carpinteria, CA); CD5 (Labvision/Neomarkers, Fremont, CA); CD10, CD23, and Bcl-2 (Novocastra/Vision Biosystem, Benton Lane, Newcastle-upon-Tyne, UK); CD15 (Becton-Dickinson Biosciences, San Jose, CA); PAX-5 (Transduction Labs, San Diego, CA); Ki-67 (Ventana, Tucson, AZ); and S100 protein (BioGenex, Fremont, CA, USA).

Specifically for this study, in cases with available paraffin-embedded tissue sections we assessed for evidence of MAPK/ERK pathway activation in the foci of Rosai-Dorfman disease using antibodies for cyclin D1 (dilution 1:350; Labvision/Neomarkers) and phospho-p44/ 42 MAPK (Thr202/Tyr204) (D13.14.4E) and p-ERK (dilution 1:300, Cell Signaling, Danvers, MA). The latter antibody allows for assessment of nuclear and cytoplasmic phosphorylated p44 and p42 MAPK (Erk1 and Erk2). Results for cyclin D1 and p-ERK were considered positive if staining was present in $>5 \%$ of Rosai-Dorfman disease histiocytes with moderate or strong intensity.

\section{Fluorescence in situ hybridization}

Fluorescence in situ hybridization (FISH) analysis to detect CCND1-IGH was performed in one case using a Vysis LSI 
$I G H / C C N D 1$ dual color, dual fusion translocation probe on interphase nuclei obtained from paraffin-embedded tissue sections, according to the manufacturer's instructions (Vysis/Abbott Laboratories, Des Plaines, IL, USA).

\section{Next-generation sequencing}

We performed amplicon-based next-generation sequencing targeting the coding regions of a panel of 146 genes that are commonly mutated in hematopoietic neoplasms using the Ion Torrent platform (Thermo Fisher Scientific, Waltham, MA, USA) on DNA extracted from micro-dissected foci of Rosai-Dorfman disease. For one patient, we were able to retrieve tissue that was not involved by Rosai-Dorfman disease or lymphoma to be used as a control. We used $20 \mathrm{ng}$ of DNA to prepare the genomic library. The genes included in the panel are as follows: $A K T 1, A K T 2, A K T 3, A L K, A R$, ARAF, ARIDIA, ATM, ATR, ATRX, AXL, BAPI, BRAF, BRCA1, BRCA2, BTK, CBL, CCND1, CCND2, CCND3, CCNE1, CDK12, CDK2, CDK4, CDK6, CDKN1B, CDKN2A,CDKN2B, CHEK1, CHEK2, CREBBP, CSF1R, CTNNB1, DDR2, EGFR, ERBB2, ERBB3, ERBB4, ERCC2, ESR1, EZH2, FANCA, FANCD2, FANCI, FBXW7, FGF19, FGF3, FGFR1, FGFR2, FGFR3, FGFR4, FLT3, FOXL2, GATA2, GNA11, GNAQ, GNAS, H3F3A, HISTIH3B, HNF1A, HRAS, IDH1, IDH2, IGF1R, JAK1, JAK2, JAK3, KDR, KIT, KNSTRN, KRAS, MAGOH, MAP2K1, MAP2K2, MAP2K4, MAPK1, MAX, MDM2, MDM4, MED12, MET, MLH1, MRE11A, MSH2, MSH6, MTOR, MYC, MYCL, MYCN, MYD88, NBN, NF1, NF2, NFE2L2, NOTCH1, NOTCH2, NOTCH3, NRAS, NTRK1, NTRK2, NTRK3, PALB2, PDGFRA, PDGFRB, PIK3CA, PIK3CB, PIK3R1, PMS, POLE, PPARG, PPP2RIA, PTCHI, PTEN, PTPN11, RACl, RAD50, RAD51, RAD51B, RAD51C, RAD51D, RAF1, RB1, RET, RHEB, RHOA, RICTOR, RNF43, ROS1, SETD2, SF3B1, SLX4, SMAD4, SMARCA4, SMARCB1, SMO, SPOP, SRC, STAT3, STK11, TERT, TOP1, TP53, $T S C 1$, TSC2, U2AF1, and XPO1. Following successful library generation and purification, DNA was used for multiplex sequencing and analyzed using the Torrent Suite and OncoSeek data pipeline.

\section{Results}

\section{Clinical features and laboratory findings}

The study group included 12 patients, five men and seven women with a median age of 46 years (range, 23-77 years). The clinicopathologic features, molecular results, treatment, and outcome are summarized in Table 1. Concomitant Rosai-Dorfman disease and lymphoma occurred in lymph nodes at the following anatomic sites: six cervical, three axillary, one inguinal, and one supraclavicular. One patient (case \#12) presented exclusively with extranodal disease involving skin of the upper chest. Ann Arbor stage for lymphoma was available in 10 patients: two $(20 \%)$ stage I, four $(40 \%)$ stage II, three (30\%) stage III, and one (10\%) stage IV. At time of initial presentation, five (42\%) patients had B-symptoms. All patients underwent excisional biopsy, which was the first specimen diagnostic of lymphoma.

In six patients, a complete blood cell count at time of diagnosis was available. One patient (case \#4) presented with leukopenia (median white blood cell count, $5.8 \times 10^{3} /$ $\mu \mathrm{l}$; range, $2.8-8.8 \times 10^{3} / \mu \mathrm{l}$; reference range, $4-11 \times 10^{3} / \mu \mathrm{l}$ ); one patient (case \#1) had lymphopenia (median absolute lymphocyte count, $1.51 \times 10^{3} / \mu \mathrm{l}$; range, $0.96-2.13 \times 10^{3} / \mu \mathrm{l}$; reference range, $1-4.8 \times 10^{3} / \mu \mathrm{l}$ ) and two patients (cases \#2 and \#5) had eosinophilia (median absolute eosinophil count, $0.26 \times 10^{3} / \mu \mathrm{l}$; range, $0.06-2.4 \times 10^{3} / \mu \mathrm{l}$; reference range, $\left.0.08-0.7 \times 10^{3} / \mu \mathrm{l}\right)$. Two patients (cases \#4 and \#7) presented with anemia (median hemoglobin, $13.4 \mathrm{~g} / \mathrm{dl}$; range, $11.2-14.7 \mathrm{~g} / \mathrm{dl}$; reference range, $14-18 \mathrm{~g} / \mathrm{dl}$ for men and $12-16 \mathrm{~g} / \mathrm{dl}$ for women). The platelet count was normal in every patient (median, $224.5 \times 10^{3} / \mu \mathrm{l}$; range, $173-341 \times$ $10^{3} / \mu \mathrm{l}$; reference range, $\left.140-440 \times 10^{3} / \mu \mathrm{l}\right)$. Serum lactate dehydrogenase was elevated in two of six patients (median, 643.5 IU/l; range, 393-944 IU/l; reference range, 313-618 IU/l) and $\beta 2$-microglobulin levels were elevated in all five patients with available data (median, $3.8 \mathrm{mg} / 1$; range, 2-7 $\mathrm{mg} / \mathrm{l}$; reference range, $0.7-1.8 \mathrm{mg} / \mathrm{l}$ ).

Among 11 patients with available medical history, two had adult-onset diabetes mellitus. None of these patients had a personal or family history of other autoimmune diseases, immunodeficiency, or signs or symptoms of inherited conditions, including those associated with Rosai-Dorfman disease, such as H or Faisalabad syndrome or autoimmune lymphoproliferative syndrome type Ia.

\section{Histologic features}

The involved lymph nodes grossly ranged from 1 to $4 \mathrm{~cm}$ (median, $2.5 \mathrm{~cm}$ ); the skin lesion was $2 \mathrm{~cm}$. The lymphomas included six $(50 \%)$ cases of nodular lymphocyte predominant Hodgkin lymphoma; four (33\%) cases of classical Hodgkin lymphoma; and one case each of small lymphocytic lymphoma and extranodal marginal zone lymphoma of mucosa-associated lymphoid tissue (MALT) involving the skin.

Microscopically, the cases of nodular lymphocyte predominant Hodgkin lymphoma included five cases with predominantly pattern A (Fig. 1) and one case of predominantly pattern E. The four cases of classical Hodgkin lymphoma included two nodular sclerosis, one mixed cellularity ' (Fig. 2), and one lymphocyte-rich classical. The case of small lymphocytic lymphoma showed expanded 


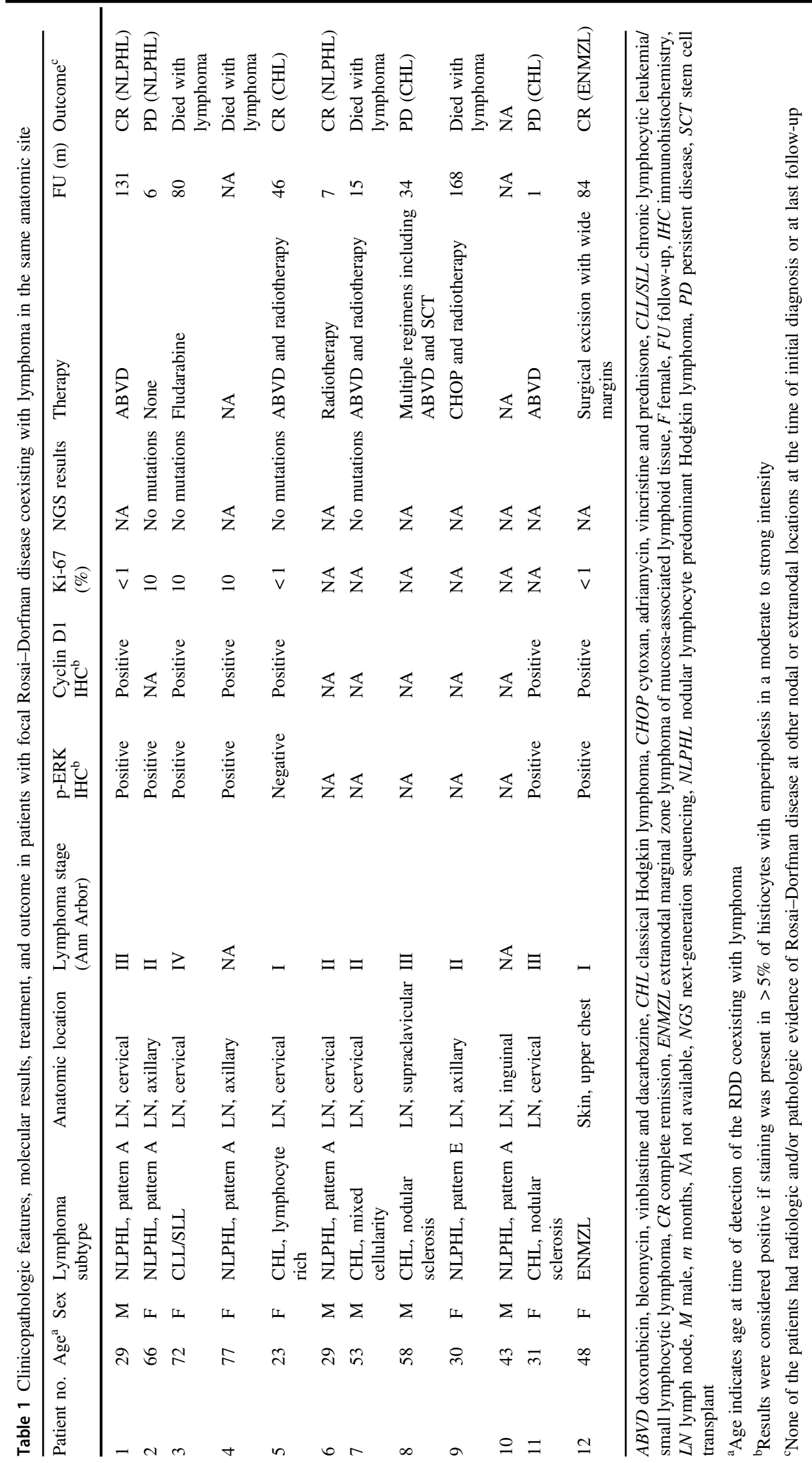



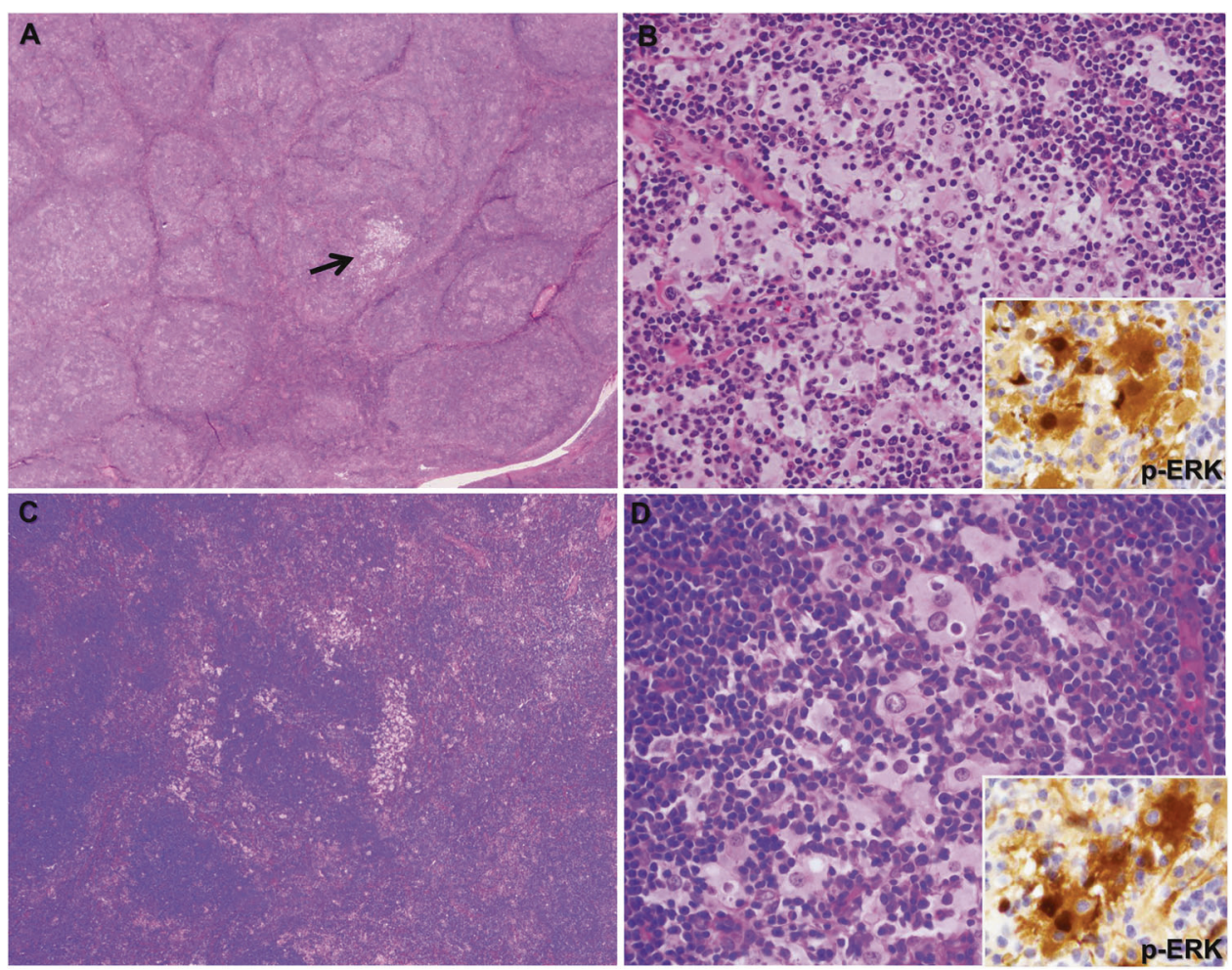

Fig. 1 Focal Rosai-Dorfman disease coexisting with nodular lymphocyte predominant Hodgkin lymphoma (cases 1 and 2). (a, case 1) Low-power view of a lymph node involved by nodular lymphocyte predominant Hodgkin lymphoma and a microscopic focus of Rosai-Dorfman disease $(20 \times$, arrow). (b, case 1) High-power magnification of histiocytes engulfing numerous leukocytes $(400 \times)$. This case is positive for p-ERK by immunohistochemistry (inset, $1000 \times$ ). (c, case 2) Low-power image of a lymph node involved by nodular lymphocyte predominant Hodgkin lymphoma and multiple microscopic clusters of Rosai-Dorfman disease surrounding lymphoid nodules $(20 \times)$. (d, case 2$)$ High-power magnification of histiocytes undergoing emperipolesis $(400 \times)$. This case is positive for p-ERK by immunohistochemistry (Inset, $1000 \times$ )

(broader than a 20x field) proliferation centers (Fig. 3), but otherwise did not show evidence of transformation and was not associated with lymphocytosis.

In addition to lymphoma, all biopsy specimens contained one to five foci (median, 2) of Rosai-Dorfman disease. The size of these foci ranged from $3 \mathrm{~mm}$ up to $20 \mathrm{~mm}$ (median, $12 \mathrm{~mm}$ ) and represented approximately $1 \%$ up to $60 \%$ of the entire composite lesion (median, 5\%). Of note, in all cases the sinuses were obliterated by lymphoma and therefore Rosai-Dorfman disease histiocytes were not present within sinuses. Instead, lesional histiocytes tended to satellite around lymphoid nodules, particularly in the cases of Hodgkin lymphoma with nodular architecture (Figs. 1, 2).

The foci of Rosai-Dorfman disease were characterized by aggregates of large histiocytes with round or oval nuclei, dispersed chromatin, visible nucleoli, and abundant pale to eosinophilic cytoplasm (Figs. 1-3). Emperipolesis of lymphocytes, plasma cells and/or granulocytes by a subset of histiocytes was identified in every case. The histiocytes were commonly mixed with plasma cells $(11 / 12 ; 92 \%)$ and granulocytes $(9 / 12 ; 75 \%)$, including neutrophils and eosinophils.
In cases of classical Hodgkin lymphoma, Rosai-Dorfman disease histiocytes were often part of the mixed cellular background wherein Reed-Sternberg or Hodgkin cells were embedded (Fig. 2e). The case of small lymphocytic lymphoma was the only case where Rosai-Dorfman disease occupied greater than half of the lymph node dimension. At low-power examination, the two processes seemed to be relatively well-delineated from each other (Fig. 3a), but at higher power the Rosai-Dorfman disease histiocytes were closely intermingled with lymphoma cells (Fig. 3c, d). None of the cases showed increased mitotic activity or areas of necrosis within the Rosai-Dorfman disease foci.

\section{Immunohistochemical assessment}

In every case, the immunohistochemical studies supported the diagnosis of lymphoma. In cases of nodular lymphocyte predominant Hodgkin lymphoma, the lymphocyte predominant (LP) cells were positive for CD20 and Bcl-6. In one case with available p-ERK and cyclin D1, the LP cells were also moderately to strongly positive for p-ERK and 
Fig. 2 Focal Rosai-Dorfman disease coexisting with classical Hodgkin lymphoma, mixed cellularity (case 8). Lymph node involved by classical Hodgkin lymphoma associated with foci of Rosai-Dorfman disease (pale areas). The abnormal histiocytes satellite around lymphoid nodules $(\mathbf{a}, 20 \times ; \mathbf{b}, 100 \times$; c, $200 \times$ ). Medium- and highpower magnification show histiocytes with emperipolesis intimately associated with Hodgkin Reed-Sternberg cells (d, $400 \times$; e, $1000 \times$ ). Immunostain for S100 protein highlights Rosai-Dorfman histiocytes and provides contrast with negatively outlined lymphocytes and Hodgkin Reed-Sternberg cells (f, arrows, $1000 \times$ )
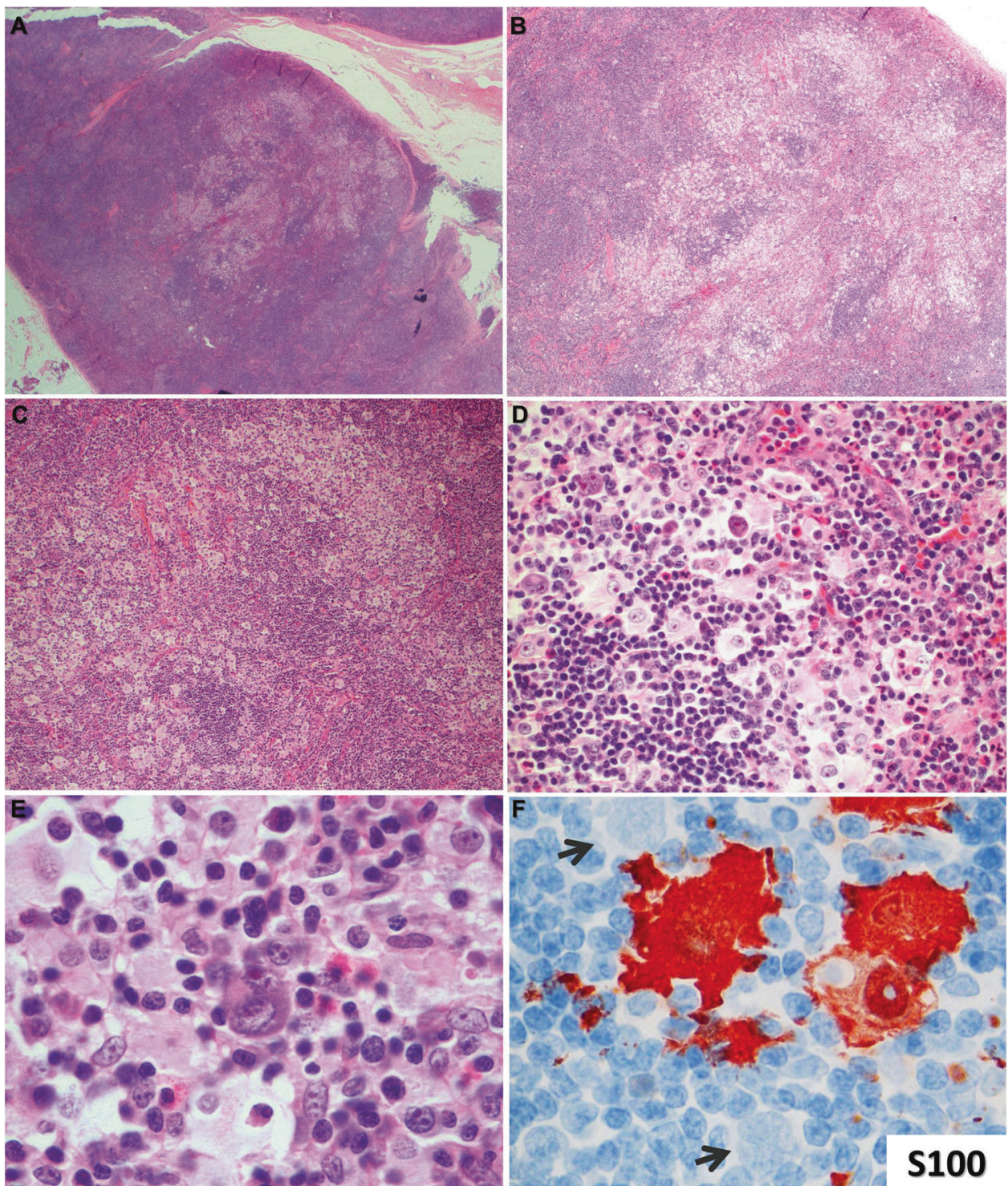

weakly positive for cyclin D1 with a nuclear staining pattern. In the remaining cases, the LP cells were negative for p-ERK and cyclin D1. In cases of classical Hodgkin lymphoma, the neoplastic cells were positive for CD15, CD30, MUM1/IRF4, and Pax-5. In all cases of Hodgkin lymphoma, the neoplastic cells were negative for T-cell antigens and CD45/LCA and those with available data were negative for p-ERK and cyclin D1. The case of small lymphocytic lymphoma was positive for CD5, CD23, and Bcl-2 and negative for cyclin D1, and the case of extranodal marginal zone lymphoma was positive for B-cell antigens and $\mathrm{Bcl}-2$, but negative for T-cell antigens (including CD5) and Bcl-6.

In every case, the Rosai-Dorfman disease histiocytes were strongly positive for $\mathrm{S} 100$ protein and negative for CD1a and CD163. CD68 was expressed variably. The Rosai-Dorfman disease histiocytes were positive for p-ERK in six of seven (86\%) cases assessed. In all positive cases, there was moderate to strong expression of p-ERK in $>50 \%$ of the Rosai-Dorfman disease histiocytes in a nuclear and cytoplasmic pattern (Fig. 1b, d, inset). All six cases tested for cyclin D1 showed that $>50 \%$ of Rosai-Dorfman disease histiocytes had moderate to strong nuclear positivity and a few cases showed weak cytoplasmic reactivity. FISH analysis using the $I G H / C C N D 1$ dual color dual fusion on one of the strongly cyclin D1-positive cases (case \#3) showed no evidence of CCND1 rearrangement or amplification. Five of six cases positive for cyclin D1 were also p-ERK positive (Fig. 3e, f), whereas one case was p-ERK negative. In three cases, Ki-67 showed a proliferation index of approximately $10 \%$ in the Rosai-Dorfman disease foci, whereas in three other cases the proliferation index was $<1 \%$. A summary of these findings is provided in Table 1. 
Fig. 3 Focal Rosai-Dorfman disease coexisting with small lymphocytic lymphoma in the same lymph node (case 3 ). a Note the paler quality at the sites with predominant histiocytic proliferation. Although not obvious at low magnification, Rosai-Dorfman macrophages were intermingled between the lymphoma cells, comprising more than half of the tissue $(20 \times)$. b At medium power, numerous histiocytes with emperipolesis and occasional giant cells are seen admixed with the lymphoma cells $(400 \times)$. c Higher magnification shows numerous histiocytes engulfing lymphocytes and plasma cells $(400 \times)$. d Immunostain for $\mathrm{S} 100$ protein is positive in histiocytes and negative in the remainder of cells $(400 \times)$. e Immunostain for $\mathrm{p}$-ERK is positive in the Rosai-Dorfman disease histiocytes $(500 \times)$. f Immunostain for cyclin D1 is strongly positive in the nucleus and weakly positive in the cytoplasm of Rosai-Dorfman disease histiocytes $(1000 \times)$
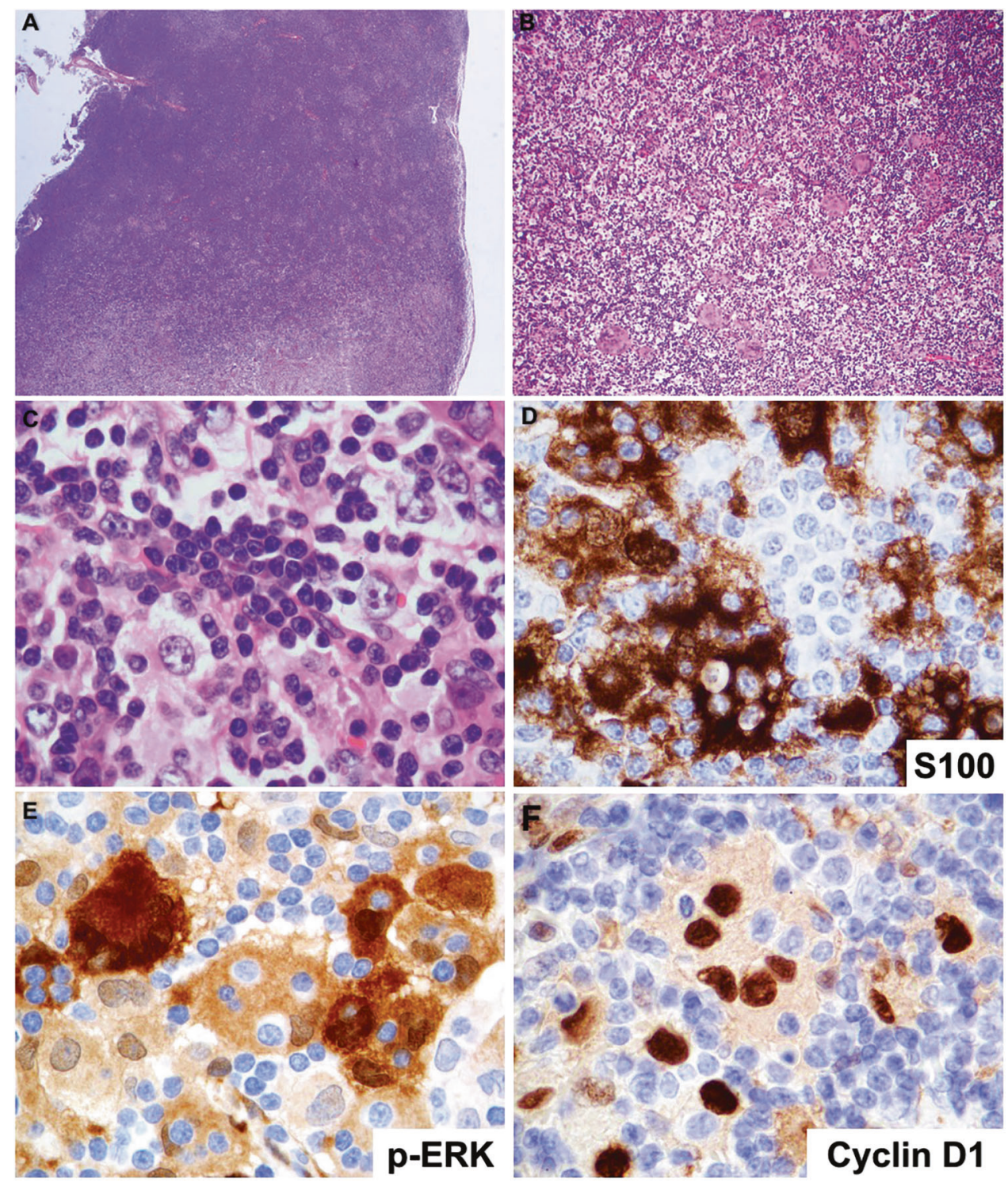

\section{Next-generation sequencing results}

DNA from micro-dissected foci of Rosai-Dorfman disease was adequate in four patients. As stated above, the 146-gene panel included genes shown previously to be relevant to the pathogenesis of histiocytic disorders, such as $A R A F, B R A F$, CCND1, ERBB1, ERBB2, ERBB3, KRAS, MAP2K1, NRAS, $P I K 3 C A, S R C$, and SMAD4. No gene mutations were detected (Table 1).

\section{Patient outcomes}

Treatment information was available for 10 patients. Seven patients received chemotherapy for their lymphoma (chemotherapy regimens are listed in Table 1); three of these patients also received additional radiation therapy and one underwent stem cell transplantation after chemotherapy.
One patient received radiotherapy only, one patient underwent wide surgical excision only, and one patient was untreated due to comorbidities associated with uncontrolled diabetes mellitus. In two patients, treatment information was not available. None of the 10 patients with available data received treatment specific for Rosai-Dorfman disease, nor did this diagnosis impact treatment decisions.

Clinical follow-up data were available for 11 patients (Table 1). One patient (case \# 10) was lost to follow-up. With a median follow-up of 40 months (range, 1-168 months), four patients remained in clinical remission, three patients were alive with persistent lymphoma, and four patients died with lymphoma. None of these patients showed Rosai-Dorfman disease at other nodal or extranodal sites in subsequent pathology specimens or had suspicious follow-up radiologic images. 


\section{Discussion}

The association between Rosai-Dorfman disease and lymphoma is rare. In this setting, Rosai-Dorfman disease can precede, coincide with, or follow the diagnosis of lymphoma and the two pathologic processes may involve the same or different anatomic sites [4-12, 14-17]. In cases of Rosai-Dorfman disease and lymphoma involving the same anatomic site, the histiocytic infiltrate is most often an incidental finding $[4,5,10-12,15]$. Although recent discoveries have highlighted the role of MAPK/ERK pathway activating mutations in the pathogenesis of Rosai-Dorfman disease [19, 21, 22, 25-27], the underlying biology of Rosai-Dorfman disease coexisting with lymphoma has not yet been elucidated.

The MAPK/ERK cascade is an important regulatory mechanism of cell function that is dysregulated in different solid tumors and hematologic diseases, the latter including many types of histiocytic proliferation including Rosai-Dorfman disease [19, 21, 22, 25-31]. Under physiologic conditions, MAPK/ERK signaling is activated by a wide variety of extracellular and intracellular stimuli, including hormones, growth factors and to a lesser extent inflammatory cytokines and environmental stresses $[29,30,32]$. Essentially, activation of MAPK/ERK signaling encompasses a three-level cytoplasmic protein kinase circuit that concludes with the phosphorylation and activation of the serine/threonine kinases ERK 1/2. Activated ERK shifts to the nucleus where it coordinates the activity of various proteins including components of the cell cycle machinery [28-30].

In this study, we analyzed 12 cases of Rosai-Dorfman disease and lymphoma involving the same anatomic site. In every patient, the detection of Rosai-Dorfman disease was incidental, patients did not have evidence of Rosai-Dorfman disease at other nodal or extranodal sites, and follow-up of these patients was unremarkable. These findings are in accord with those of others [4, 5, 10-12, 15]. In line with this benign behavior, in four cases we did not identify mutations in any of 146 genes examined $[19,25,33,34]$. Nevertheless, we did identify evidence of MAPK/ERK pathway activation including p-ERK expression in the nucleus and cytoplasm of Rosai-Dorfman disease histiocytes, as well as nuclear expression of cyclin D1 in Rosai-Dorfman histiocytes.

Cyclin D1 constitutes one of the most important downstream targets through which the MAPK/ERK pathway exerts its effects in cell cycle progression. For a cell to undergo G1 to S-phase transition, newly synthesized cyclin D1 must dimerize with the cyclin-dependent kinases 4 and 6 (CDK 4/6). Subsequently, the cyclin D1-CDK4/6 complex promotes passage through G1-phase and entry into S-phase by phosphorylation of retinoblastoma protein [35-37].
Activation of the MAPK/ERK pathway has been shown to be both necessary and sufficient for transcriptional induction of CCND1 [35-37]. Moreover, activated ERK1/2 has been shown to facilitate the formation of cyclin D1-CDK4 complexes in fibroblasts [38]. Expression of cyclin D1 has been shown by others to be a useful surrogate marker of MAPK/ERK pathway activation in other neoplasms [39-41]. The data we present here suggest ERK-induced cyclin D1 upregulation within the histiocytes of focal Rosai-Dorfman disease.

Because no mutations were identified in this small cohort, other molecular mechanisms of MAPK/ERK pathway activation must be considered. The most frequent kinase-activating genetic lesions that have been linked to histiocytic proliferations are mutations; nevertheless, other recently described mechanisms include gene fusions involving BRAF, ALK, NTRK1, and ETV3-NCOA2 [25, 34]. Whereas the technology applied in this study is able to detect insertions, deletions, and point mutations, it does not detect gene fusions. Additionally, we cannot rule out mutations in other genes not included in our next-generation sequencing panel.

Other possible mechanisms unrelated to mutations that may underlie the development of Rosai-Dorfman disease in this setting are related to the microenvironment induced by the associated lymphoma. In this case series, the two most frequent lymphomas associated with Rosai-Dorfman disease were nodular lymphocyte predominant Hodgkin lymphoma and classical Hodgkin lymphoma, in aggregate representing about $80 \%$ of all cases. Others have shown a similar high frequency of Hodgkin lymphomas coexisting with Rosai-Dorfman disease in the same anatomic site. A summary of previously reported cases is provided in Table 2 [4, 5, 10-12, 15]. In Hodgkin lymphomas, the neoplastic cells are embedded in an inflammatory milieu where stimulatory molecules, including growth factors (e.g., platelet-derived growth factor-alpha and transforming growth factor- $\beta$ ), cytokines and chemokines (e.g., IL-3, IL6 , IL-7, IL-9, IL-13, IL-21, and tumor necrosis factor- $\alpha$ ) that are produced by both tumor and non-neoplastic cells can generate a niche for neoplastic growth and survival [42-44]. Therefore, it seems reasonable to hypothesize that a focal Rosai-Dorfman histiocytic proliferation might be the result of an autocrine and/or paracrine loop driving MAPK/ERK signaling. In this setting, focal Rosai-Dorfman disease is analogous to cases of Langerhans cell histiocytosis coexisting with lymphoma in the same site. In a recent study of seven cases of Langerhans cell histiocytosis detected incidentally in association with lymphoma, no gene mutations were identified although a subset of these cases showed p-ERK expression [45]. Notably, the lymphoma coexisting with Langerhans cell histiocytosis was classical Hodgkin lymphoma in many cases and mechanisms of 


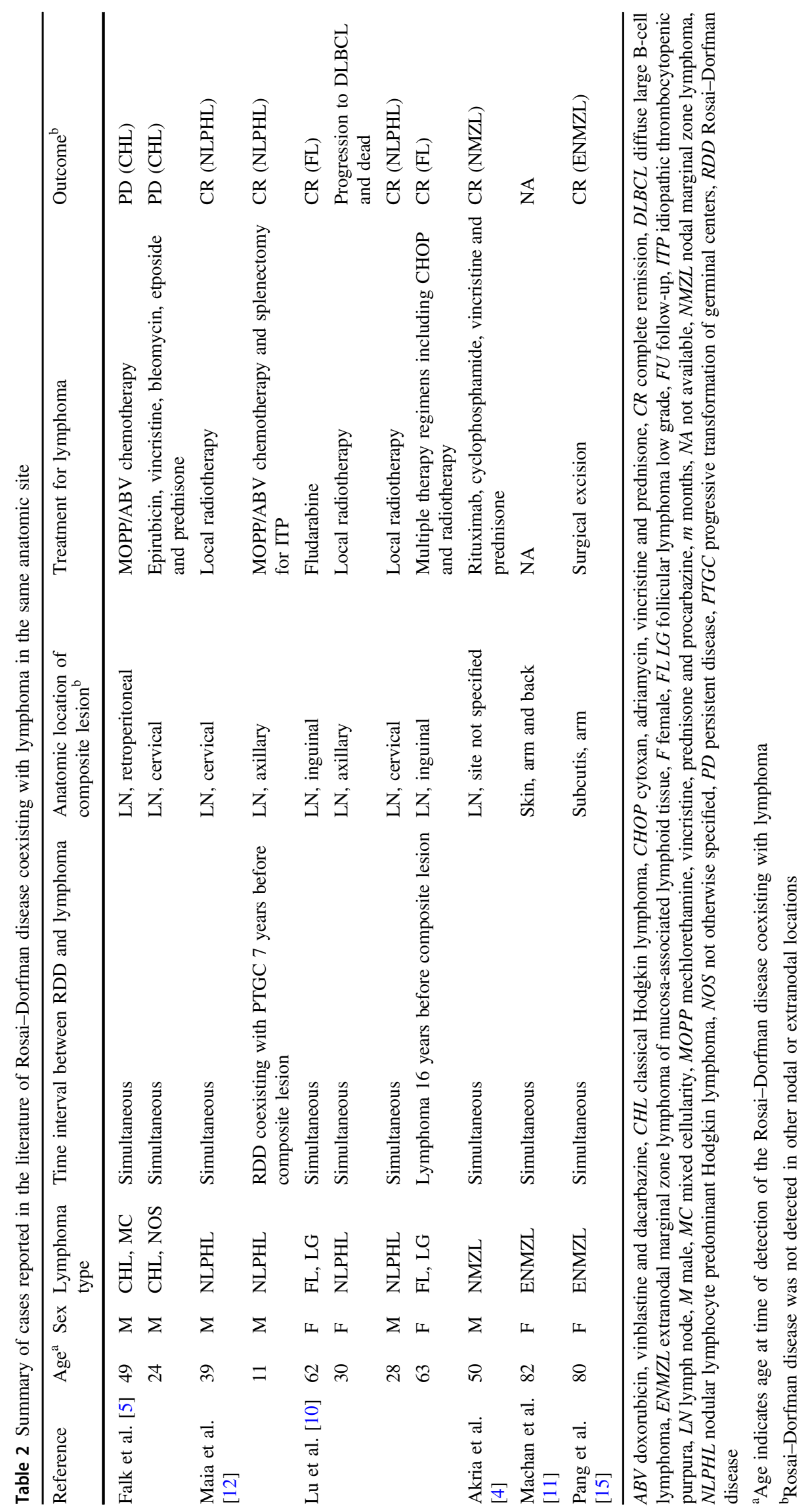


MAPK/ERK pathway activation in incidental Langerhans cell histiocytosis and focal Rosai-Dorfman disease may be similar.

The findings in this study also raise the issue of the best designation for focal Rosai-Dorfman disease associated with lymphoma. Are these cases truly Rosai-Dorfman disease or only a morphologic mimic? The absence of bulky lymphadenopathy or extranodal disease, the incidental detection and clinically benign behavior, and the absence of gene mutations in four cases all suggest that these lesions are a morphologic mimic of Rosai-Dorfman disease. In addition, Abla et al. [2] have suggested that focal Rosai-Dorfman representing $<10 \%$ of a pathology specimen is a reactive process, a criterion that many of the cases we report did not meet. On the other side of this question, four cases are likely an insufficient number to claim that gene mutations do not occur in focal Rosai-Dorfman disease and the gene panel we used was somewhat limited. In addition, the Rosai-Dorfman histiocytes formed welldefined aggregates, showed emperipolesis, were associated with a characteristic plasmacytic infiltrate, and all cases assessed showed evidence of MAPK/ERK pathway activation. These data support the designation of Rosai-Dorfman disease and are in line with the designation of these lesions as Rosai-Dorfman disease in the past, by Dorfman and others $[11,12,14]$. Based on this study, we cannot definitively answer the question we propose, but we can state that the pathogenesis of focal Rosai-Dorfman disease appears to be different from that of usual cases of Rosai-Dorfman disease.

In summary, the detection of Rosai-Dorfman disease associated with lymphoma is clinically benign in patients who are treated for their lymphoma. Similar to usual cases of Rosai-Dorfman disease, the histiocytes in focal Rosai-Dorfman disease associated with lymphoma show evidence of MAPK/ERK pathway activation, but we did not identify gene mutations in four cases assessed. It seems likely that the pathogenesis of focal Rosai-Dorfman disease associated with lymphoma differs from usual cases of Rosai-Dorfman disease. As has been suggested by others, it is possible that MAPK/ERK pathway activation is induced by molecules (e.g., cytokines) that are prevalent in the associated lymphoma microenvironment.

Acknowledgements We thank Ronald C. Cason and Jawad Manekia for technical assistance in the performance of the next-generation sequencing studies.

\section{Compliance with ethical standards}

Conflict of interest The authors declare that they have no conflict of interest.

\section{References}

1. Rosai J, Dorfman RF. Sinus histiocytosis with massive lymphadenopathy. A newly recognized benign clinicopathological entity. Arch Pathol. 1969;87:63-70.

2. Abla O, Jacobsen E, Picarsic J, et al. Consensus recommendations for the diagnosis and clinical management of Rosai-DorfmanDestombes disease. Blood. 2018;131:2877-90.

3. Humble JG, Jayne WH, Pulvertaft RJ. Biological interaction between lymphocytes and other cells. $\mathrm{Br} \mathrm{J}$ Haematol. 1956;2:283-94.

4. Akria L, Sonkin V, Braester A, et al. Rare coexistence of RosaiDorfman disease and nodal marginal zone lymphoma complicated by severe life-threatening autoimmune hemolytic anemia. Leuk Lymphoma. 2013;54:1553-6.

5. Falk S, Stutte HJ, Frizzera G. Hodgkin's disease and sinus histiocytosis with massive lymphadenopathy-like changes. Histopathology. 1991;19:221-4.

6. Foucar E, Rosai J, Dorfman RF, et al. Immunologic abnormalities and their significance in sinus histiocytosis with massive lymphadenopathy. Am J Clin Pathol. 1984;82:515-25.

7. Koduru PR, Susin M, Kolitz JE, et al. Morphological, ultrastructural, and genetic characterization of an unusual T-cell lymphoma in a patient with sinus histiocytosis with massive lymphadenopathy. Am J Hematol. 1995;48:192-200.

8. Krzemieniecki K, Pawlicki M, Marganska K, et al. The RosaiDorfman syndrome in a 17-year-old woman with transformation into high-grade lymphoma. A rare disease presentation. Ann Oncol. 1996;7:977.

9. Lossos IS, Okon E, Bogomolski-Yahalom V, et al. Sinus histiocytosis with massive lymphadenopathy (Rosai-Dorfman disease): report of a patient with isolated renotesticular involvement after cure of non-Hodgkin's lymphoma. Ann Hematol. 1997;74:41-4.

10. Lu D, Estalilla OC, Manning JT, et al. Sinus histiocytosis with massive lymphadenopathy and malignant lymphoma involving the same lymph node: a report of four cases and review of the literature. Mod Pathol. 2000;13:414-9.

11. Machan S, Medina C, Rodriguez-Pinilla SM, et al. Primary cutaneous marginal IgG4 lymphoma and Rosai-Dorfman's disease coexisting in several lesions of the same patient. Am J Dermatopathol. 2015;37:413-8.

12. Maia DM, Dorfman RF. Focal changes of sinus histiocytosis with massive lymphadenopathy (Rosai-Dorfman disease) associated with nodular lymphocyte predominant Hodgkin's disease. Hum Pathol. 1995;26:1378-82.

13. Menzel C, Hamscho N, Dobert N, et al. PET imaging of RosaiDorfman disease: correlation with histopathology and ex-vivo beta-imaging. Arch Dermatol Res. 2003;295:280-3.

14. Moore JC, Zhao X, Nelson EL. Concomitant sinus histiocytosis with massive lymphadenopathy (Rosai-Dorfman Disease) and diffuse large B-cell lymphoma: a case report. J Med Case Rep. 2008;2:70.

15. Pang CS, Grier DD, Beaty MW. Concomitant occurrence of sinus histiocytosis with massive lymphadenopathy and nodal marginal zone lymphoma. Arch Pathol Lab Med. 2011;135:390-3.

16. Rangwala AF, Zinterhofer LJ, Nyi KM, et al. Sinus histiocytosis with massive lymphadenopathy and malignant lymphoma. An unreported association. Cancer . 1990;65:999-1002.

17. Shoda H, Oka T, Inoue M, et al. Sinus histiocytosis with massive lymphadenopathy associated with malignant lymphoma. Intern Med. 2004;43:741-5.

18. Wu W, Cao L, Li Y, et al. Primary splenic diffuse large B-cell lymphoma in a patient with thymus Rosai-Dorfman disease. Am J Med Sci. 2012;344:155-9. 
19. Garces S, Medeiros LJ, Patel KP, et al. Mutually exclusive recurrent KRAS and MAP2K1 mutations in Rosai-Dorfman disease. Mod Pathol. 2017;30:1367-77.

20. Shanmugam V, Margolskee E, Kluk M, et al. Rosai-Dorfman disease harboring an activating KRAS K117N missense mutation. Head Neck Pathol. 2016;10:394-9.

21. Jacobsen E, Shanmugam V, Jagannathan J. Rosai-Dorfman disease with activating KRAS mutation - response to cobimetinib. $\mathrm{N}$ Engl J Med. 2017;377:2398-9.

22. Matter MS, Bihl M, Juskevicius D, et al. Is Rosai-Dorfman disease a reactive process? Detection of a MAP2K1 L115V mutation in a case of Rosai-Dorfman disease. Virchows Arch. 2017;471:545-7.

23. Ragotte RJ, Dhanrajani A, Pleydell-Pearce J, et al. The importance of considering monogenic causes of autoimmunity: a somatic mutation in KRAS causing pediatric Rosai-Dorfman syndrome and systemic lupus erythematosus. Clin Immunol. 2017;175:143-6.

24. Yabe M, Medeiros LJ, Wang SA, et al. Distinguishing between hepatosplenic T-cell lymphoma and gammadelta T-cell large granular lymphocytic leukemia: a clinicopathologic, immunophenotypic, and molecular analysis. Am J Surg Pathol. 2017;41:82-93.

25. Diamond EL, Durham BH, Haroche J, et al. Diverse and targetable kinase alterations drive histiocytic neoplasms. Cancer Discov. 2016;6:154-65.

26. Ozkaya N, Dogan A, Abdel-Wahab O. Identification and targeting of kinase alterations in histiocytic neoplasms. Hematol Oncol Clin North Am. 2017;31:705-19.

27. Piris MA, Aguirregoicoa E, Montes-Moreno S, et al. Castleman disease and Rosai-Dorfman disease. Semin Diagn Pathol. 2018;35:44-53.

28. Lewis TS, Shapiro PS, Ahn NG. Signal transduction through MAP kinase cascades. Adv Cancer Res. 1998;74:49-139.

29. Roux PP, Blenis J. ERK and p38 MAPK-activated protein kinases: a family of protein kinases with diverse biological functions. Microbiol Mol Biol Rev. 2004;68:320-44.

30. Krishna M, Narang H. The complexity of mitogen-activated protein kinases (MAPKs) made simple. Cell Mol Life Sci. 2008;65:3525-44.

31. Burotto M, Chiou VL, Lee JM, et al. The MAPK pathway across different malignancies: a new perspective. Cancer. 2014;120: 3446-56.

32. Whitmarsh AJ. Regulation of gene transcription by mitogenactivated protein kinase signaling pathways. Biochim Biophys Acta. 2007;1773:1285-98.
33. Gatalica Z, Bilalovic N, Palazzo JP, et al. Disseminated histiocytoses biomarkers beyond BRAFV600E: frequent expression of PD-L1. Oncotarget. 2015;6:19819-25.

34. Durham BH. Molecular characterization of the histiocytoses: neoplasia of dendritic cells and macrophages. Semin Cell Dev Biol. 2018 [e-pub ahead of print].

35. Chambard JC, Lefloch R, Pouyssegur J, et al. ERK implication in cell cycle regulation. Biochim Biophys Acta. 2007;1773:1299-310.

36. Meloche S, Pouyssegur J. The ERK1/2 mitogen-activated protein kinase pathway as a master regulator of the G1- to S-phase transition. Oncogene. 2007;26:3227-39.

37. Sherr CJ, McCormick F. The RB and p53 pathways in cancer. Cancer Cell. 2002;2:103-12.

38. Cheng M, Sexl V, Sherr CJ, et al. Assembly of cyclin Ddependent kinase and titration of p27Kip1 regulated by mitogenactivated protein kinase kinase (MEK1). Proc Natl Acad Sci USA. 1998;95:1091-6.

39. Miranda RN, Briggs RC, Kinney MC, et al. Immunohistochemical detection of cyclin D1 using optimized conditions is highly specific for mantle cell lymphoma and hairy cell leukemia. Mod Pathol. 2000;13:1308-14.

40. Shanmugam V, Craig JW, Hornick JL, et al. Cyclin D1 Is expressed in neoplastic cells of Langerhans cell histiocytosis but not reactive Langerhans cell proliferations. Am J Surg Pathol. 2017;41:1390-6.

41. Smalley KS, Lioni M, Dalla Palma M, et al. Increased cyclin D1 expression can mediate BRAF inhibitor resistance in BRAF V600E-mutated melanomas. Mol Cancer Ther. 2008;7: 2876-83.

42. Cattaruzza L, Gloghini A, Olivo K, et al. Functional coexpression of Interleukin (IL)-7 and its receptor (IL-7R) on Hodgkin and Reed-Sternberg cells: involvement of IL-7 in tumor cell growth and microenvironmental interactions of Hodgkin's lymphoma. Int J Cancer. 2009;125:1092-101.

43. Skinnider BF, Mak TW. The role of cytokines in classical Hodgkin lymphoma. Blood. 2002;99:4283-97.

44. Aldinucci D, Olivo K, Lorenzon D, et al. The role of interleukin-3 in classical Hodgkin's disease. Leuk Lymphoma. 2005;46:303-11.

45. Pina-Oviedo S, Medeiros LJ, Li S, et al. Langerhans cell histiocytosis associated with lymphoma: an incidental finding that is not associated with BRAF or MAP2K1 mutations. Mod Pathol. 2017;30:734-44. 